\title{
Multiplicity of positive solutions of superlinear semi-positone singular Neumann problems
}

\author{
Qiuyue $\mathrm{Li}^{1 *}$, Fuzhong Cong ${ }^{1}$, Zhe $\mathrm{Li}^{2}$ and Jinkai LV${ }^{1}$
}

“Correspondence: liqy609@163.com 'Department of Foundation Courses, Aviation University of Airforce, Renmin Street 7855, Changchun, 130012, China Full list of author information is available at the end of the article

\begin{abstract}
Introduction: Neumann boundary value problems have been studied by many authors. We are mainly interested in the semi-positone case. This paper deals with the existence and multiplicity of positive solutions of a superlinear semi-positone singular Neumann boundary value problem.

Preliminaries: The proof of our main results relies on a nonlinear alternative of Leray-Schauder type, the method of upper and lower solutions and on a well-known fixed point theorem in cones.
\end{abstract}

Main results: We obtained the existence of at least two different positive solutions.

Keywords: positive solutions; superlinear; semi-positone; singular; Neumann problem

\section{Introduction}

We will be concerned with the existence and multiplicity of positive solutions of the superlinear singular Neumann boundary value problem in the semi-positone case

$$
\left\{\begin{array}{l}
-\left(p(x) u^{\prime}\right)^{\prime}+q(x) u=g(x, u), \quad x \in I=[0,1] \\
u^{\prime}(0)=0, \quad u^{\prime}(1)=0 .
\end{array}\right.
$$

Here the type of perturbations $g(x, u)$ may be singular near $u=0$ and $g(x, u)$ is superlinear near $u=+\infty$. From the physical point of view, $g(x, u)$ has an attractive singularity near $u=0$ if

$$
\lim _{u \rightarrow 0^{+}} g(x, u)=+\infty \quad \text { uniformly in } x
$$

and the superlinearity of $g(x, u)$ means that

$$
\lim _{u \rightarrow+\infty} g(x, u) / u=+\infty \quad \text { uniformly in } x
$$

By the semi-positone case of (1.1), we mean that $g(x, u)$ may change sign and satisfies $F(x, u)=g(x, u)+M \geq 0$ where $M>0$ is a constant.

\section{Springer}

(0) 2014 Li et al.; licensee Springer. This is an Open Access article distributed under the terms of the Creative Commons Attribution License (http://creativecommons.org/licenses/by/4.0), which permits unrestricted use, distribution, and reproduction in any medium, provided the original work is properly credited. 
It is well known that the existence of positive solutions of boundary value problems has been studied by many authors in [1-6] and references therein. They mainly considered the case of $p(x) \equiv 1$ and $q(x) \equiv 0$. In [7], the authors studied positive solutions of Neumann boundary problems of second order impulsive differential equations in the positone case, based on a nonlinear alternative principle of Leray-Schauder type and a well-known fixed point theorem in cones. This paper attempts to study the existence and multiplicity of positive solutions of second order superlinear singular Neumann boundary value problems in the semi-positone case. The techniques we employ here involve a nonlinear result of Leray-Schauder, the well-known fixed point theorem in cones and the method of upper and lower solutions. We prove that problem (1.1) has at least two different positive solutions. Moreover, we do not take the restrictions $p(x) \equiv 1$ or $q(x) \equiv 0$.

Throughout this paper, we assume that the perturbed part $g(x, u)$ satisfies the following hypotheses:

$\left(\mathrm{H}_{1}\right) g(x, u) \in C\left(I \times R^{+}, R^{+}\right), p(x) \in C^{1}(I), q(x) \in C(I), p(x)>0, q(x)>0$.

$\left(\mathrm{H}_{2}\right)$ There exists a constant $M>0$ such that $F(x, u)=g(x, u)+M \geq 0$ for all $x \in I$ and $u \in(0, \infty)$.

In Section 2, we perform a study of the sign of the Green's function of the corresponding linear problems

$$
\left\{\begin{array}{l}
-\left(p(x) u^{\prime}\right)^{\prime}+q(x) u=h(x), \quad x \in I, \\
u^{\prime}(0)=0, \quad u^{\prime}(1)=0 .
\end{array}\right.
$$

In detail, we construct the Green's function $G(x, y)$ and give a sufficient condition to ensure $G(x, y)$ is positive. This fact is crucial for our arguments. We denote

$$
A=\min _{(x, y) \in I \times I} G(x, y), \quad B=\max _{(x, y) \in I \times I} G(x, y), \quad \sigma=A / B .
$$

We also use $\omega(x)$ to denote the unique solution of $(1.2)$ with $h(x)=1, \omega(x)=\int_{0}^{1} G(x, y) d y$. In Section 3, we state and prove the main results of this paper.

\section{Preliminaries}

For the reader's convenience we introduce some results of Green's functions. Let $Q=I \times I$, $Q_{1}=\{(x, y) \in Q \mid 0 \leq x \leq y \leq 1\}, Q_{2}=\{(x, y) \in Q \mid 0 \leq y \leq x \leq 1\}$.

Considering the homogeneous boundary value problem

$$
\left\{\begin{array}{l}
-\left(p(x) u^{\prime}\right)^{\prime}+q(x) u=0, \quad x \in I \\
u^{\prime}(0)=0, \quad u^{\prime}(1)=0
\end{array}\right.
$$

and let $G(x, y)$ be the Green's function of problem (2.1). Then $G(x, y)$ can be written as

$$
G(x, y)= \begin{cases}\frac{m(x) n(y)}{\omega}, & (x, y) \in Q_{1}, \\ \frac{m(y) n(x)}{\omega}, & (x, y) \in Q_{2},\end{cases}
$$

where $m$ and $n$ are linearly independent, and $m, n$ and $\omega$ satisfy the following lemma. 
Lemma 2.1 [7] Suppose that $\left(\mathrm{H}_{1}\right)$ holds and problem (2.1) has only zero solution, then there exist two functions $m(x)$ and $n(x)$ satisfying:

(i) $m(x) \in C^{2}(I, R)$ is increasing and $m(x)>0, x \in I$;

(ii) $n(x) \in C^{2}(I, R)$ is decreasing and $n(x)>0, x \in I$;

(iii) $L m \equiv-\left(p(x) m^{\prime}\right)^{\prime}+q(x) m=0, m(0)=1, m^{\prime}(0)=0$;

(iv) $L n \equiv-\left(p(x) n^{\prime}\right)^{\prime}+q(x) n=0, n(1)=1, n^{\prime}(1)=0$;

(v) $\omega \equiv p(x)\left(m^{\prime}(x) n(x)-m(x) n^{\prime}(x)\right)$ is a positive constant.

Lemma 2.2 [7] The Green's function $G(x, y)$ defined by (2.2) has the following properties:

(i) $G(x, y)$ is continuous in $Q$;

(ii) $G(x, y)$ is symmetrical on $Q$;

(iii) $G(x, y)$ has continuous partial derivatives on $Q_{1}, Q_{2}$;

(iv) For each fixed $y \in I, G(x, y)$ satisfies $L G(x, y)=0$ for $x \neq y, x \in I$. Moreover, $G_{x}^{\prime}(0, y)=G_{x}^{\prime}(1, y)=0$ for $y \in(0,1)$.

(v) For $x=y, G_{x}^{\prime}$ has discontinuity point of the first kind, and

$$
G_{x}^{\prime}(y+0, y)-G_{x}^{\prime}(y-0, y)=-\frac{1}{p(y)}, \quad y \in(0,1) .
$$

Lemma 2.3 [8] Suppose that conditions in Lemma 2.1 hold and $h: I \rightarrow R$ is continuous. Then the problem

$$
\left\{\begin{array}{l}
-\left(p(x) u^{\prime}\right)^{\prime}+q(x) u=h(x), \quad x \in I, \\
u^{\prime}(0)=0, \quad u^{\prime}(1)=0,
\end{array}\right.
$$

has a unique solution, which can be written as

$$
u(x)=\int_{0}^{1} G(x, y) h(y) d y .
$$

Next we state the theorem of fixed points in cones, which will be used in Section 3.

Theorem 2.1 [9] Let $X$ be a Banach space and $K(\subset X)$ be a cone. Assume that $\Omega_{1}, \Omega_{2}$ are open subsets of $X$ with $0 \in \Omega_{1}, \bar{\Omega}_{1} \subset \Omega_{2}$, and let

$$
T: K \cap\left(\bar{\Omega}_{2} \backslash \Omega_{1}\right) \rightarrow K
$$

be a continuous and compact operator such that either

(i) $\|T u\| \geq\|u\|, u \in K \cap \partial \Omega_{1}$ and $\|T u\| \leq\|u\|, u \in K \cap \partial \Omega_{2}$; or

(ii) $\|T u\| \leq\|u\|, u \in K \cap \partial \Omega_{1}$ and $\|T u\| \geq\|u\|, u \in K \cap \partial \Omega_{2}$.

Then $T$ has a fixed point in $K \cap\left(\bar{\Omega}_{2} \backslash \Omega_{1}\right)$.

In applications below, we take $X=C(I)$ with the supremum norm $\|\cdot\|$ and define

$$
K=\left\{u \in X: u(x) \geq 0 \text { and } \min _{x \in I} u(x) \geq \sigma\|u\|\right\} .
$$


One may readily verify that $K$ is a cone in $X$. Now suppose that $F: I \times R \rightarrow[0, \infty)$ is continuous and define an operator $T: X \rightarrow X$ by

$$
(T u)(x)=\int_{0}^{1} G(x, y) F(y, u(y)) d y
$$

for $u \in X$ and $x \in[0,1]$.

Lemma 2.4 $T$ is well defined and maps $X$ into K. Moreover, $T$ is continuous and completely continuous.

\section{Main results}

In this section we establish the existence and multiplicity of positive solutions to (1.1). Since we are mainly interested in the attractive-superlinear nonlinearities $g(x, u)$ in the semi-positone case, we assume that the hypotheses of the following theorem are satisfied.

Theorem 3.1 Suppose that $\left(\mathrm{H}_{1}\right)$ and $\left(\mathrm{H}_{2}\right)$ hold. Furthermore, assume the following:

$\left(\mathrm{H}_{3}\right)$ There exist continuous, non-negative functions $f(u)$ and $g(u)$ such that

$$
F(x, u)=g(x, u)+M \leq f(u)+h(u) \quad \text { for all }(x, u) \in I \times(0, \infty),
$$

and $f(u)>0$ is non-increasing and $h(u) / f(u)$ is non-decreasing in $u \in(0, \infty)$.

$\left(\mathrm{H}_{4}\right)$ There exists $r>\frac{M\|\omega\|}{\sigma}$ such that $\frac{r}{f(\sigma r-M\|\omega\|)\left\{1+\frac{h(r)}{f(r)}\right\}}>\|\omega\|$.

$\left(\mathrm{H}_{5}\right)$ There exists a constant $A>M, \varepsilon>0$ such that

$$
F(x, u) \geq A, \quad f(u)>A \quad \text { for all }(x, u) \in I \times(0, \varepsilon] .
$$

Then problem (1.1) has at least one positive solution $v \in C(I)$ with $0<\|v+M \omega\|<r$.

Before we present the proof of Theorem 3.1, we state and prove some facts.

First, it is easy to see that we can take $c>0$ and $n_{0}>1$ such that

$$
\begin{aligned}
& c\|\omega\|<\min \left\{\varepsilon, \frac{A-M}{\|q\|}\right\}, \\
& \frac{1}{n_{0}}<\min \left\{\varepsilon, \frac{\varepsilon}{M}, c \sigma\|\omega\|, \sigma r-M\|\omega\|\right\} .
\end{aligned}
$$

Lemma 3.1 Suppose that $\left(\mathrm{H}_{1}\right)-\left(\mathrm{H}_{5}\right)$ hold, then $\alpha(x)=(M+c) \omega(x)$ is a strict lower solution to the problem

$$
\left\{\begin{array}{l}
-\left(p(x) u^{\prime}\right)^{\prime}+q(x) u=F_{n}(x, u-M \omega(x)), \quad x \in I, n>n_{0}, \\
u^{\prime}(0)=0, \quad u^{\prime}(1)=0
\end{array}\right.
$$

where $F_{n}(x, u)=F\left(x, \max \left\{u, \frac{1}{n}\right\}\right),(x, u) \in I \times R$.

Proof It is easy to see that $\alpha^{\prime}(0)=(M+c) \omega^{\prime}(0)=0$ and $\alpha^{\prime}(1)=(M+c) \omega^{\prime}(1)=0$. 
Since $\alpha(x)-M \omega(x)=c \omega(x) \geq c \sigma\|\omega\|>\frac{1}{n_{0}} \geq \frac{1}{n}$, and using (3.1), we have $\varepsilon>\alpha(x)-$ $M \omega(x)=c \omega(x) \geq \frac{1}{n}>0$.

By assumption $\left(\mathrm{H}_{5}\right)$, we have

$$
F_{n}(x, \alpha(x)-M \omega(x))>A, \quad \forall n>n_{0} .
$$

This implies that $\alpha(x)$ is a strict lower solution to (3.3).

Lemma 3.2 Suppose that $\left(\mathrm{H}_{1}\right)-\left(\mathrm{H}_{5}\right)$ hold. Then the problem

$$
\left\{\begin{array}{l}
-\left(p(x) u^{\prime}\right)^{\prime}+q(x) u=f_{n}(u-M \omega(x))\left(1+\frac{h(r)}{f(r)}\right), \quad x \in I \\
u^{\prime}(0)=0, \quad u^{\prime}(1)=0
\end{array}\right.
$$

has at least one positive solution $\beta_{n}(x)$ with $\left\|\beta_{n}\right\|<r$.

Proof The existence is proved using the Leray-Schauder alternative principle together with a truncation technique.

Since $\left(\mathrm{H}_{4}\right)$ holds, we have

$$
\|\omega\| f(\sigma r-M\|\omega\|)(1+h(r) / f(r))<r .
$$

Consider the family of problems

$$
\left\{\begin{array}{l}
-\left(p(x) u^{\prime}\right)^{\prime}+q(x) u=\lambda f_{n}(u-M \omega(x))\left(1+\frac{h(r)}{f(r)}\right), \quad x \in I, \\
u^{\prime}(0)=0, \quad u^{\prime}(1)=0
\end{array}\right.
$$

where $\lambda \in I$ and $f_{n}(u)=f(\max \{u, 1 / n\}),(x, u) \in I \times R . f_{n}(u)$ is non-increasing.

Problem (3.5) is equivalent to the following fixed point problem in $C[0,1]$

$$
\beta=\lambda T_{n} \beta
$$

where $T_{n}$ is defined by

$$
T_{n}(\beta(x))=\int_{0}^{1} G(x, y) f_{n}(\beta(y)-M \omega(y))(1+h(r) / f(r)) d y .
$$

We claim that any fixed point $\beta$ of (3.6) for any $\lambda \in[0,1]$ must satisfy $\|\beta\| \neq r$. Otherwise, assume that $\beta$ is a solution of (3.6) for some $\lambda \in[0,1]$ such that $\|\beta\|=r$. Note that $f_{n}(x, u) \geq 0$. By Lemma 3.1, for all $x, \beta(x)-M \omega(x) \geq \sigma r-M\|\omega\| \geq 1 / n$. Hence, for all $x$,

$$
\beta(x)-M \omega(x) \geq 1 / n \quad \text { and } \quad \beta(x)-M \omega(x) \geq \sigma r-M\|\omega\| .
$$

Then we have, for all $x$,

$$
\begin{aligned}
\beta(x) & =\lambda \int_{0}^{1} G(x, y) f_{n}(\beta(y)-M \omega(y))\left(1+\frac{h(r)}{f(r)}\right) d y \\
& \leq \int_{0}^{1} G(x, y) f(\beta(y)-M \omega(y))\left(1+\frac{h(r)}{f(r)}\right) d y
\end{aligned}
$$




$$
\begin{aligned}
& \leq \int_{0}^{1} G(x, y) f(\sigma r-M\|\omega\|)(1+h(r) / f(r)) d y \\
& \leq\|\omega\| f(\sigma r-M\|\omega\|)(1+h(r) / f(r)) .
\end{aligned}
$$

Therefore,

$$
r=\|\beta\| \leq\|\omega\| f(\sigma r-M\|\omega\|)(1+h(r) / f(r))<r .
$$

This is a contradiction and the claim is proved.

From this claim, the nonlinear alternative of Leray-Schauder guarantees that problem (3.6) (with $\lambda=1$ ) has a fixed point, denoted by $\beta_{n}$, in $B_{r}$, i.e., problem (3.4) has a positive solution $\beta_{n}$ with $\left\|\beta_{n}\right\|<r$. (In fact, it is easy to see that $\beta_{n}(x) \geq 1 / n$ with $\left\|\beta_{n}\right\| \neq r$.)

Lemma 3.3 Suppose that $\left(\mathrm{H}_{1}\right)-\left(\mathrm{H}_{5}\right)$ hold, then $\beta_{n}(x)$ is an upper solution of problem (3.3).

Proof By Lemma 3.2 we know that $\beta_{n}(x)$ is a solution to equation (3.4).

If $\beta_{n}(x)-M \omega(x) \geq \frac{1}{n}$, then

$$
\begin{aligned}
F_{n}\left(x, \beta_{n}(x)-M \omega(x)\right) & =F\left(x, \beta_{n}(x)-M \omega(x)\right) \\
& \leq f\left(\beta_{n}(x)-M \omega(x)\right)\left(1+\frac{h\left(\beta_{n}(x)-M \omega(x)\right)}{f\left(\beta_{n}(x)-M \omega(x)\right)}\right) \\
& \leq f_{n}\left(\beta_{n}(x)-M \omega(x)\right)\left(1+\frac{h(r)}{f(r)}\right) .
\end{aligned}
$$

If $\beta_{n}(x)-M \omega(x) \leq \frac{1}{n}$, then

$$
\begin{aligned}
F_{n}\left(x, \beta_{n}(x)-M \omega(x)\right) & =F\left(x, \frac{1}{n}\right) \leq f\left(\frac{1}{n}\right)\left(1+\frac{h\left(\frac{1}{n}\right)}{f\left(\frac{1}{n}\right)}\right) \\
& \leq f_{n}\left(\beta_{n}(x)-M \omega(x)\right)\left(1+\frac{h(r)}{f(r)}\right) .
\end{aligned}
$$

Since $\beta_{n}^{\prime}(0)=\beta_{n}^{\prime}(1)=0$, we have

$$
\left\{\begin{array}{l}
-\left(p(x) \beta_{n}^{\prime}(x)\right)^{\prime}+q(x) \beta_{n}(x) \geq F_{n}\left(x, \beta_{n}(x)-M \omega(x)\right), \quad x \in I, \\
\beta_{n}^{\prime}(0)=0, \quad \beta_{n}^{\prime}(1)=0 .
\end{array}\right.
$$

This implies that $\beta_{n}(x)$ is an upper solution of problem (3.3).

Lemma 3.4 Suppose that $\left(\mathrm{H}_{1}\right)-\left(\mathrm{H}_{5}\right)$ hold, then $\beta_{n}(x) \geq \alpha(x)\left(n>n_{0}\right)$.

Proof Let $z(x)=\alpha(x)-\beta_{n}(x)$, we will prove $z(x) \leq 0$. If this is not true for $n>n_{0}$, there exists $x_{0} \in[0,1]$ such that $z\left(x_{0}\right)=\max z(x)>0, z^{\prime}\left(x_{0}\right)=0, z^{\prime \prime}\left(x_{0}\right) \leq 0$. Then $\left(p\left(x_{0}\right) z^{\prime}\left(x_{0}\right)\right)^{\prime} \leq 0$. 
Since $\alpha\left(x_{0}\right)-M \omega\left(x_{0}\right)=c \omega\left(x_{0}\right) \geq c \sigma\|\omega\|>\frac{1}{n_{0}} \geq \frac{1}{n}, \alpha\left(x_{0}\right)-M \omega\left(x_{0}\right) \leq c\|\omega\|<\varepsilon$, and $f_{n}(u)$ is non-increasing, we have

$$
\begin{aligned}
f_{n}\left(\beta\left(x_{0}\right)-M \omega\left(x_{0}\right)\right) & \geq f_{n}\left(\alpha\left(x_{0}\right)-M \omega\left(x_{0}\right)\right) \\
& =f\left(\alpha\left(x_{0}\right)-M \omega\left(x_{0}\right)\right) \\
& >A
\end{aligned}
$$

and

$$
\begin{aligned}
-\left(p\left(x_{0}\right) z^{\prime}\left(x_{0}\right)\right)^{\prime}+q\left(x_{0}\right) z\left(x_{0}\right) & =M+c-f_{n}\left(\beta_{n}\left(x_{0}\right)-M \omega\left(x_{0}\right)\right)\left(1+\frac{h(r)}{f(r)}\right) \\
& \leq M+c-f_{n}\left(\alpha\left(x_{0}\right)-M \omega\left(x_{0}\right)\right)\left(1+\frac{h(r)}{f(r)}\right) \\
& \leq M+c-A\left(1+\frac{h(r)}{f(r)}\right)<0 .
\end{aligned}
$$

This is a contradiction and completes the proof of Lemma 3.4.

Proof of Theorem 3.1 To show (1.1) has a positive solution, we will show

$$
\left\{\begin{array}{l}
-\left(p(x) u^{\prime}\right)^{\prime}+q(x) u=F(x, u(x)-M \omega(x)), \quad x \in I \\
u^{\prime}(0)=u^{\prime}(1)=0
\end{array}\right.
$$

has a solution $u \in C(I), u(x)>M \omega(x), x \in I$.

If this is true, then $v(x)=u(x)-M \omega(x)$ is a positive solution of (1.1) since

$$
\begin{aligned}
-\left(p(x) v^{\prime}\right)^{\prime}+q(x) v & =-\left(p(x) u^{\prime}(x)-p(x) M \omega^{\prime}(x)\right)^{\prime}+q(x) u(x)-M q(x) \omega(x) \\
& =-\left(p(x) u^{\prime}(x)\right)^{\prime}+q(x) u(x)-M \\
& =F(x, u(x)-M \omega(x))-M \\
& =g(x, u(x)-M \omega(x)) \\
& =g(x, v(x)) .
\end{aligned}
$$

As a result, we will only concentrate our study on (3.14).

By Lemmas 3.1-3.4 and the upper and lower solutions method, we know that (3.3) has a solution $u_{n}$ with $(M+c) \omega(x)=\alpha(x) \leq u_{n}(x) \leq \beta_{n}(x)<r$. Thus we have $u_{n}(x)-M \omega(x) \geq$ $c \sigma\|\omega\|, u_{n}(x) \leq \beta_{n}(x)<r$.

By the fact that $u_{n}$ is a bounded and equi-continuous family on [0,1], the Arzela-Ascoli theorem guarantees that $\left\{u_{n}\right\}_{n \in N_{0}}$ has a subsequence $\left\{u_{n_{k}}\right\}_{k \in N}$, which converges uniformly on $[0,1]$ to a function $u \in C[0,1]$. Then $u$ satisfies $u(x)-M \omega(x) \geq c \sigma\|\omega\|, u(x)<r$ for all $x$. Moreover, $u_{n_{k}}$ satisfies the integral equation

$$
u_{n_{k}}(x)=\int_{0}^{1} G(x, y) F\left(y, u_{n_{k}}(y)-M \omega(y)\right) d y
$$


Letting $k \rightarrow \infty$, we arrive at

$$
u(x)=\int_{0}^{1} G(x, y) F(y, u(y)-M \omega(y)) d y
$$

where the uniform continuity of $F(x, u(x)-M \omega(x))$ on $[0,1] \times[c \sigma\|\omega\|, r]$ is used. Therefore, $u$ is a positive solution of (3.14).

Finally, it is not difficult to show that $\|u\|<r$. Assume otherwise: note that $F(x, u) \geq 0$. By Lemma 2.4, for all $x, u(x) \geq 1 / n$ and $r \geq u(x)-M \omega(x) \geq \sigma r-M\|\omega\| \geq 1 / n$. Hence, for all $x$,

$$
u(x)-M \omega(x) \geq 1 / n \quad \text { and } \quad r \geq u(x)-M \omega(x) \geq \sigma r-M\|\omega\| .
$$

Then we have for all $x$,

$$
\begin{aligned}
u(x) & =\int_{0}^{1} G(x, y) F(y, u(y)-M \omega(y)) d y \\
& \leq \int_{0}^{1} G(x, y) f(u(y)-M \omega(y))\left(1+\frac{h(u(y)-M \omega(y))}{f(u(y)-M \omega(y))}\right) d y \\
& \leq \int_{0}^{1} G(x, y) f(\sigma r-M\|\omega\|)(1+h(r) / f(r)) d y \\
& \leq\|\omega\| f(\sigma r-M\|\omega\|)(1+h(r) / f(r)) .
\end{aligned}
$$

Therefore,

$$
r=\|u\| \leq\|\omega\| f(\sigma r-M\|\omega\|)(1+h(r) / f(r)) .
$$

This is a contradiction and completes the proof of Theorem 3.1.

Corollary 3.1 Let us consider the following boundary value problem

$$
\left\{\begin{array}{l}
-\left(p(x) u^{\prime}\right)^{\prime}+q(x) u=\mu\left(u^{-\alpha}+u^{\beta}+k(x)\right), \quad x \in I \\
u^{\prime}(0)=u^{\prime}(1)=0
\end{array}\right.
$$

where $\alpha>0, \beta>0$ and $k:[0,1] \rightarrow R$ is continuous, $\mu>0$ is chosen such that

$$
\mu<\sup _{u \in\left(\frac{M\|\omega\|}{\sigma}, \infty\right)} \frac{u(\sigma u-M\|\omega\|)^{\alpha}}{\|\omega\|\left\{1+2 H u^{\alpha}+u^{\alpha+\beta}\right\}}
$$

here $H=\|k\|$. Then problem (3.17) has a positive solution $u \in C[0,1]$.

Proof We will apply Theorem 3.1 with $M=\mu H$ and

$$
f(u)=f_{1}(u)=\mu u^{-\alpha}, \quad h(u)=\mu\left(u^{\beta}+2 H\right), \quad h_{1}(u)=\mu u^{\beta} .
$$

Clearly, $\left(\mathrm{H}_{1}\right)-\left(\mathrm{H}_{3}\right)$ and $\left(\mathrm{H}_{5}\right)$ are satisfied. 
Set

$$
T(u)=\frac{u(\sigma u-M\|\omega\|)^{\alpha}}{\|\omega\|\left\{1+2 H u^{\alpha}+u^{\alpha+\beta}\right\}}, \quad u \in\left(\frac{M\|\omega\|}{\sigma},+\infty\right) .
$$

Since $T\left(\frac{M\|\omega\|}{\sigma}\right)=0, T(\infty)=0$, then there exists $r \in\left(\frac{M\|\omega\|}{\sigma}, \infty\right)$ such that

$$
T(r)=\sup _{u \in\left(\frac{M\|\omega\|}{\sigma}, \infty\right)} \frac{u(\sigma u-M\|\omega\|)^{\alpha}}{\|\omega\|\left\{1+2 H u^{\alpha}+u^{\alpha+\beta}\right\}} .
$$

This implies that there exists $r \in\left(\frac{M\|\omega\|}{\sigma}, \infty\right)$ such that $\mu<\frac{r(\sigma r-M\|\omega\|)^{\alpha}}{\|\omega\|\left\{1+r^{\alpha+\beta}+2 H r^{\alpha}\right\}}$, so $\left(\mathrm{H}_{4}\right)$ is satisfied.

Since $\beta>1$. Thus all the conditions of Theorem 3.1 are satisfied, so the existence is guaranteed.

Next we will find another positive solution to problem (1.1) by using Theorem 2.1.

Theorem 3.2 Suppose that conditions $\left(\mathrm{H}_{1}\right)-\left(\mathrm{H}_{5}\right)$ hold. In addition, it is assumed that the following two conditions are satisfied:

$\left(\mathrm{H}_{6}\right) \quad F(x, u)=g(x, u)+M \geq f_{1}(u)+h_{1}(u)$ for some continuous non-negative functions $f_{1}(u)$ and $h_{1}(u)$ with the properties that $f_{1}(u)>0$ is non-increasing and $h_{1}(u) / f_{1}(u)$ is nondecreasing.

$\left(\mathrm{H}_{7}\right)$ There exists $R>r$ such that $\frac{R}{\sigma f_{1}(R)\left\{1+\frac{h_{1}(\sigma R-M\|\omega\|)}{f_{1}(\sigma R-M\|\omega\|)}\right\}}<\|\omega\|$.

Then, besides the solution $u$ constructed in Theorem 3.1, problem (1.1) has another positive solution $\tilde{v} \in C[0,1]$ with $r<\|\tilde{v}+M \omega\| \leq R$.

Proof To show (1.1) has a positive solution, we will show (3.14) has a solution $\tilde{u} \in C[0,1]$ with $\tilde{u}(x)>M \omega(x)$ for $x \in[0,1]$ and $r \leq\|\tilde{u}\| \leq R$.

Let $X=C[0,1]$ and $K$ be a cone in $X$ defined by (2.5). Let

$$
\Omega_{r}=\{\tilde{u} \in U:\|\tilde{u}\|<r\}, \quad \Omega_{R}=\{\tilde{u} \in X:\|\tilde{u}\|<R\}
$$

and define the operator $T: K \cap\left(\bar{\Omega}_{R} \backslash \Omega_{r}\right) \rightarrow K$ by

$$
(T \tilde{u})(x)=\int_{0}^{1} G(x, y) F(y, \tilde{u}(y)-M \omega(y)) d y, \quad 0 \leq x \leq 1,
$$

where $G(x, y)$ is as in $(2.2)$.

For each $\tilde{u} \in K \cap\left(\bar{\Omega}_{R} \backslash \Omega_{r}\right) r \leq\|\tilde{u}\| \leq R$, we have $0<\sigma r-M\|\omega\| \leq \tilde{u}(x)-M \omega(x) \leq R$. Since $F:[0,1] \times[\sigma r-M\|\omega\|, R] \rightarrow[0, \infty)$ is continuous, it follows from Lemma 2.4 that the operator $T: K \cap\left(\bar{\Omega}_{R} \backslash \Omega_{r}\right) \rightarrow K$ is well defined, is continuous and completely continuous.

First we show 
In fact, if $\tilde{u} \in K \cap \partial \Omega_{r}$, then $\|\tilde{u}\|=r$ and $\tilde{u}(x) \geq \sigma r>M\|\omega\|$ for $x \in I$. So we have

$$
\begin{aligned}
(T \tilde{u})(x) & =\int_{0}^{1} G(x, y) F(y, \tilde{u}(y)-M \omega(y)) d y \\
& \leq \int_{0}^{1} G(x, y) f(\tilde{u}(y)-M \omega(y))\left\{1+\frac{h(\tilde{u}(y)-M \omega(y))}{f(\tilde{u}(y)-M \omega(y))}\right\} d y \\
& \leq \int_{0}^{1} G(x, y) f(\sigma r-M\|\omega\|)\left\{1+\frac{h(r)}{f(r)}\right\} d y \\
& =\omega(x) f(\sigma r-M\|\omega\|)\left\{1+\frac{h(r)}{f(r)}\right\} \\
& \leq\|\omega\| f(\sigma r-M\|\omega\|)\left\{1+\frac{h(r)}{f(r)}\right\} \\
& <r=\|\tilde{u}\| .
\end{aligned}
$$

This implies $\|T \tilde{u}\|<\|\tilde{u}\|$, i.e., (3.20) holds.

Next we show

$$
\|T \tilde{u}\|>\|\tilde{u}\| \quad \text { for } \tilde{u} \in K \cap \partial \Omega_{R} .
$$

To see this, let $\tilde{u} \in K \cap \partial \Omega_{R}$, then $\|\tilde{u}\|=R$ and $\tilde{u}(x) \geq \sigma R>M\|\omega\|$ for $x \in I$. As a result, it follows from $\left(\mathrm{H}_{6}\right)$ and $\left(\mathrm{H}_{7}\right)$ that, for $x \in I$,

$$
\begin{aligned}
(T \tilde{u})(x) & =\int_{0}^{1} G(x, y) F(y, \tilde{u}(y)-M \omega(y)) d y \\
& \geq \int_{0}^{1} G(x, y) f_{1}(\tilde{u}(y)-M \omega(y))\left\{1+\frac{h_{1}(\tilde{u}(y)-M \omega(y))}{f_{1}(\tilde{u}(y)-M \omega(y))}\right\} d y \\
& \geq \int_{0}^{1} G(x, y) f_{1}(R)\left\{1+\frac{h_{1}(\sigma R-M\|\omega\|)}{f_{1}(\sigma R-M\|\omega\|)}\right\} d y \\
& =\omega(x) f_{1}(R)\left\{1+\frac{h_{1}(\sigma R-M\|\omega\|)}{f_{1}(\sigma R-M\|\omega\|)}\right\} \\
& \geq \sigma\|\omega\| f_{1}(R)\left\{1+\frac{h_{1}(\sigma R-M\|\omega\|)}{f_{1}(\sigma R-M\|\omega\|)}\right\} \\
& >R=\|\tilde{u}\| .
\end{aligned}
$$

Now (3.20), (3.21) and Theorem 2.1 guarantee that $T$ has a fixed point $\tilde{u} \in K \cap\left(\bar{\Omega}_{R} \backslash \Omega_{r}\right)$ with $r \leq\|\tilde{u}\| \leq R$. Clearly, this $\tilde{u}$ is a positive solution of (3.14). This completes the proof of Theorem 3.2.

Let us consider again example (3.17) in Corollary 3.1 for the superlinear case, i.e., $\alpha>0$, $\beta>1$ and $k:[0,1] \rightarrow R$ is continuous, $\mu>0$ is chosen such that (3.18) holds, here $H=\|k\|$. Then problem (3.17) has a positive solution $\tilde{u} \in C[0,1]$. Clearly, $\left(\mathrm{H}_{1}\right)-\left(\mathrm{H}_{6}\right)$ are satisfied.

Since $\beta>1$, then $\left(\mathrm{H}_{7}\right)$ is satisfied for $R$ large enough because when $R \rightarrow \infty$,

$$
\frac{R}{\sigma f_{1}(R)\left\{1+\frac{h_{1}(\sigma R-M\|\omega\|)}{f_{1}(\sigma R-M\|\omega\|)}\right\}}=\frac{R^{\alpha+1}}{\sigma \mu\left(1+(\sigma R-M\|\omega\|)^{\alpha+\beta}\right)} \rightarrow 0 .
$$


Thus all the conditions of Theorem 3.2 are satisfied, so the existence is guaranteed.

Corollary 3.2 Assume that $\alpha>0, \beta>1$ and $k: I \rightarrow R$ is continuous, $\mu>0$ is chosen such that (3.18) holds. Take $H=\|k\|$. Then problem (3.17) has at least two different positive solutions.

\section{Competing interests}

The authors declare that they have no competing interests.

\section{Authors' contributions}

All authors contributed equally to the writing of this paper. All authors read and approved the final manuscript.

\section{Author details}

'Department of Foundation Courses, Aviation University of Airforce, Renmin Street 7855, Changchun, 130012, China.

${ }^{2}$ Department of Aviation Mechanical Engineering, Aviation University of Airforce, Nanhu Road 2222, Changchun, 130012, China

\section{Acknowledgements}

The authors express their thanks to the referee for his valuable suggestions. The work was supported by the National Natural Science Foundation of China (No: 11171350).

Received: 5 February 2014 Accepted: 12 September 2014 Published online: 08 October 2014

\section{References}

1. Hwang, B, Lee, S, Kim, Y: Existence of an unbounded branch of the set of solutions for Neumann problems involving the $p(x)$-Laplacian. Bound. Value Probl. 2014, Article ID92 (2014)

2. Chu, J, Jiang, D: Multiplicity of positive solutions to second order differential equations with Neumann boundary conditions. Appl. Math. 32, 203-303 (2005)

3. Dong, Y: A Neumann problem at resonance with the nonlinearity restricted in one direction. Nonlinear Anal. 51 , 739-747 (2002)

4. Li, X, Jiang, D: Optimal existence theory for single and multiple positive solutions to second order Neumann boundary value problems. Indian J. Pure Appl. Math. 35, 573-586 (2004)

5. Sun, J, Li, W: Multiple positive solutions to second order Neumann boundary value problems. Appl. Math. Comput. $146,187-194(2003)$

6. Lian, H, Zhao, J, Agarwal, R: Upper and lower solution method for nth-order BVPs on an infinite interval. Bound. Value Probl. 2014, Article ID100 (2014)

7. Li, Q, Cong, F, Jang, D: Multiplicity of positive solutions to second order Neumann boundary value problems with impulse actions. Appl. Math. Comput. 206, 810-817 (2008)

8. Guo, K, Sun, J, Liu, Z: Nonlinear Ordinary Differential Equations Functional Technologies. Shan Dong Science Technology, Shan Dong (1995)

9. Deimling, K: Nonlinear Functional Analysis. Springer, New York (1985)

doi:10.1186/s13661-014-0217-0

Cite this article as: $L i$ et al.: Multiplicity of positive solutions of superlinear semi-positone singular Neumann problems. Boundary Value Problems 2014 2014:217.

\section{Submit your manuscript to a SpringerOpen ${ }^{\circ}$ journal and benefit from:}

- Convenient online submission

- Rigorous peer review

- Immediate publication on acceptance

- Open access: articles freely available online

- High visibility within the field

- Retaining the copyright to your article 